\title{
PROYECTO DEL ATLAS LINGÜÍSTICO Y ETNOGRA- FICO DE LAS ISLAS CANARIAS
}

Intronucción. Breve referencia a La Situación de las ifablas de Canarias.

Las hablas de Canarias no son un dialecto, al menos lo que solemos entender por dialecto ${ }^{1}$. $\mathrm{Ni}$ uno solo de sus rasgos fonéticos es privativamente suyo; ni su léxico se diferencia de los otros hispánicos en medida que haga falta la independencia idiomática; ni su sintaxis y su morfología son exclusivas. Pertenece a ese gran complejo lingüístico que podríamos llamar hablas hispánicas meridionales y en el que cabrían el extremeño meridional, el andaluz, el murciano y, teniendo en cuenta algunas cuestiones desconocidas por la lingüística peninsular, el español de América. Otras veces se ha hablado de español atlántico ${ }^{2}$ para salvar ciertas dificultades geográficas, pero en tal denominación no cabe la totalidad de rasgos fonéticos que debemos considerar. No insistimos de momento en una nomenclatura que no matiza la cuestión, aunque no la pueda resolver definitivamente, porque las soluciones dependen - aún- de parcelas de nuestra ignorancia. En lo que sí quiero insistir es en el falseamiento -deliberado o inconsciente--- de esa realidad española que es el español de Canarias. Cuando en 1951 se publica una bibliografía de los estudios de filología románica en Europa y América ${ }^{3}$ se organizó el volumen de una manera harto extraña: aunque la bibliografía se urdenaba unas veces por naciones, otras se siguió la agrupación por dominios lingüísticos, mientras que, en algunas, el criterio preferido era el del lugar donde trabajaron los investigadores o donde imprimieron sus traba-

1 Vid. Manuel Alvar, Hacia los conceptos de lengua, dialectos y hablas, NRFH, $\mathrm{XV}, 1961$, pp. 51-60.

2 Diego Cataláx, Génesis del español atlántico. Ondas varias a través del océano. Facultad de Filosofía y Letras. Universidad de La Laguna, 1958; aunque se trata de una comunicación leída en Río de Janeiro en agosto de 1959; El çȩeo-zezeo al comenzar la expansión atlâutica de Castilla. BFit. XVI, 1956-1957 (publ. 1958), pp .306-334.

- Os estudos de linguística românica na Europa e na América desde 1939. Coimbra, 1951. (El volumen está organizado por el Prof. M. de Paiva Boléo). 
jos (practicando en estos casos un estrecho patriotismo). De los conjuntos yacionales, se apartó el habla de Canarias, con lo que las Islas no podían ser incluídas en la bibliografía porque si no cupieron en el país llamado España, mucho menos se podían insertar en una bibliografía de Europa y América. Desde una estricta consideración científica, las islas Canarias ciebían ordenarse con el español, del mismo modo que Madeira o Cabo Verde figuran en el portugués. Por otra parte, y esto no afecta al método, pero sí a los resultados, la filología románica no ha recibido nada - ni una sola línea-de la producción isleña, y la filología española, no demasiado. Juan Régulo, que redactó esa bibliografía, para poder decir algo de la linzüística en Canarias tuvo que empezar no en 1939, como en el volumen se anuncia, sino en el siglo xviır y aun hacer alguna incursión anterior. Quiero aclarar las cuestiones: cierto patriotismo jugó en este caso una carta pueril, y no ganó ninguna baza, sino que la bibliografía--desde un punto de vista científico-se descabaló. Sin embargo, este criterio nos sirve para poner -ahora-- las cosas en su punto: los trabajos de M. Steffen ${ }^{4}$, de J. Pérez. Vidal ${ }^{5}$ o de J. Régulo Pérez ${ }^{6}$ son dignos de encomio por su exactitud y sus aciertos; los de Alvarez Delgado ${ }^{\top}$ ilustran muchos aspectos de las lenguas prehispánicas de las islas, pero ¿ cómo comparar en número, variedad y tradición las contribuciones canarias -insisto: dignas de todo encomio- con las del dialecto leonés o las del dialecto aragonés? Pensemos que hasta el

4Vid. Otra vez el «garoé». RHI, X, 1944, pp. 39-45; El falso "guato» de Torriani. RHL, XIII, 1947, pp. 177-197; Lexicología canaria. I. RHL, XI, 1945, pp. 130-177; ib. II. RHI., XIV, 1948, pp. 137-176 y 414-452; ib. III, RHL, XVII, 1951, pp. 11-26; Problemas léxicos. RHL. IX, 1943, pp. 134-141; A propósito de un artículo de Dámaso Alonso. El saúco entre Galicia y Asturias (Nombre y superstición). VRo, XIV, 1954, pp. 204-222 (materiales insulares).

5 Cf. La medicina popular camaria. Tagoro, I, 1944, pp. 29-88; Portuguesismos en el español de Canarias. M, Can, [944, pp. 30-42; Prólogo y notas a la Colección de zoces de Sebastián de Lugo. La Laguna, 1946; Nombres de la lluria menuda en la isla de la Palma (Canarias). DRTP, V, 1949, pp. 177-199.

- Cf. Cuestionario sobre palabras y cosas de la isla de la Palma. La Laguna, 1946, aparte los estudios breves sobre problemas léxicos que incluyo en los números 809 . 812 y 817 de mi Dialectología española. "Cuadernos bibliográficos» VIII. Madrid, 1962

7 De una copiosa bibliografía entresaco: Puesto de Canarias en la investigación lingüistica. La Laguna 1941; Ecero. Notas lingüisticas sobre El Hierro (separata de la RHL, números 72-75, 1945-1946); Tabona. Notas lingiiísticas. RHL, XI, 1945, pp. 202-209; Teide. Ensayo de filología tinerfeña. La Laguna, 1945; Tamarán. MCan, números 21-22, 1947, pp. 27-50; Miscelánea guanche. I. Benahoare. Ensayos de linguiistica canaria. La Laguna, 1947; Etimología de "attegia" y sus relacionados. BFil, X, 1949, pp. 64-76; Algunos topónimos de Agaete. MCan, números 29-30, 1949, pp. $27-$ 36. 
Español de Tenerifo (1959) ${ }^{8}$ nunca se había publicado un libro sobre los aspectos románicos de la lingüística canaria; que hoy aún no se ha descrito ninguna de sus particularidades locales; que hasta 1959 no se habia precisado ia articulación de ningún sonido del español insular. De aquí que el español de Canarias necesite ser conocido, describir su fonética, inventariar su léxico, establece a la vinculación de palabras y cosas, analizar sus cambios semánticos, trazar su geografía lingüística. medir la altura social de sus fenómetios. Y todo ello, claro, sin olvidar la historia ${ }^{9}$ que nos hablará de los portingueses en las islas; esos portugueses, que ni para Torriani en 1950, ni siquiera para Camões, eran otra cosa que una nación más de Hispania. He. aqui un texto nítido de Torriani que me permito traducir: «Tenerife está joblada en su mayor parte por gentes portuguesas, las cuales aventajan en las técnicas agrícolas a his otras naciones españolas...» ${ }^{10}$.

\section{¿ARCaísmo? ¿CARÁCter perifÉri. CO DEL ESPAÑOL INSULAR?}

Cuando a ha tratado de caracterizar el español canario se ha hablado de su arcaísmo. Es más, se ha llegado a escribir que por su carácter periféricu se ha estancado sin evolucionar ${ }^{11}$. La hipótesis es falaz. La escuela lingüústica italiana, con sus dos grandes maestros G. Bertoni y M. G. Bartoli, en un breve y luminoso libro, el Breviario di neolinguistica ${ }^{12}$, estableció las cinco normas por las que se rige la lingüística espacial. Una de ellas, la de las úreas laterales (aree laterali), dice que las regiones marginales son más arcaizantes que las centrales ${ }^{13}$. Tal el caso del castellano, arcaizante respecto al francés o al italiano; innovador frente al catalán o al gallego-portugués. La hipótesis que comento ha tenido una mala aplicación a nuestras islas. En

8 Manuel Alvar, El español hablado en Tenerife. Premio "Antonio de Nebrija", 1955 del C. S. I. C. Anejo IXIX de la RFE. Madrid, 1959.

- Elías Serra, bencmérito profesor de la Universidad de La Laguna, ha dedicado su infatigable atención al estudio de la historia insular. Se deben consultar, por los muchos materiales útiles para una lingǘstica, las Fontes Rerum Canariarum, de las que ya se han impreso nueve volúmenes.

10 Edl. Wölfel, 1940, p. 158.

11 Vid. J. Alvarez Delgado, Notas sobre el español de Canarias. RD'TP, III, 1947, pp. 208-209, especialmente.

19 Parte I: Principi Generali; Parte II: Criteri Tecnici. Modena, 1928. Las normas son de M. G. Bartoli y figuran en la p. 66 del opúsculo. Seguidamente se explican $y$ comentan.

13 Página 68 y ejemplificación en las 69-70. 
primer lugar hay que distinguir un hecho histórico: las regiones de conquista (Canarias, América) y de reconquista (Andalucía, Sicilia, el Mezzogriorno italiano) no pueden identificarse con los territorios patrimoniales (Asturias, Aragón, Toscana, Isla de Francia). No pueden identificarse por la sencilla razón de que, en ellos, las estructuras tradicionales han sido barridas por gentes extrañas y al origen de estas gentes extrañas es a donde hay que vincular las nacientes tradiciones. Así, la estructura lingüística de Andalucía depende de unos hechos muy poco abstrusos: simplemente del origen occidental, central $\mathfrak{u}$ oriental de sus conquistadores y repobladores; la de Canarias, desde un punto de vista español, de las gentes andaluzas que aquí vinieron. No se olvide que de «Andalucía salieron las principales expediciones para la conquista y colonización de Canarias» ${ }^{14}$ y no se olvide que Alonso Fernández de Lugo, el primer adelantado de Tenerife, era andaluz y con él vinieron gentes de Sevilla y de Sanlúcar ${ }^{15}$ y que hay documentación que prueba cómo se concertó con gaditanos la empresa de ocupar Gran $\mathrm{Ca}$ naria ${ }^{16}$. Y hacia Sevilla iba el ideal de la que llegó a ser gran ciudad de Las Palmas: con su calle de Triana, con su nostalgia por tener una catedral que se pareciera a la de Sevilla o la de encargar unos cuadros que pudieran ser sevillanos ${ }^{\mathbf{1 7}}$.

Si las leyes de los neolingüistas tienen poca aplicación al español insular por causas históricas, bueno será remachar el clavo con otros argumentos: la situación de Canarias no se puede llamar periférica a humo de pajas pues, desde el primer viaje del Almirante, se pudo ver que las islas no eran periferia de nada, sino centro, eslabón intermedio que unía -válgame jugar con la palabreja- dos periferias: la peninsular y la de América. $Y$ las cosas duraron desde el año de gracia de 1492 hasta el de desgracia de 1898. Y lioy - por otros motivos- persisten todavía. El español de Canarias no es periférico, sino medular. Son los canarios quienes van a la periferia americana, como aquellos 2.500 colonos insulares que marcharon a Santo Domin-

14 Perez Vidal, apud. Diccionario de Lugo, p. 27.

is Vid. H. Sancho de Sopranis, Los ascendientes del Adelantado Alonso de Lugo, RHL, XV, 1949, p. 235, y A. Romeu DE Armas, Notas históricas al blasón de los Adelantados, RHL, XI, 1945, p. 434.

18 Vid. H. SANCho DE Sopranis, Miscelánea histórica canaria. RHL, XVIII, 1952, p. 40.

17 Cf. Jesús Hernández Perera, La Catedral de Santa Ana y Flandes. RHı, XVIII, 1952, pp. 442-454; IDEM, Orfebrería de Canarias. Madrid, 1955, p. 111, y Sobre los arquitectos de la Catedral de Las Palmas, 1500-157o. M Can, 73-74, 1960, página 270. 
go en una época en que la vieja Española no llegaba a los 6.000 habitantes ${ }^{18}$. Por otra parte, queda esa escurridiza e inasible cuestión de los arcaísmos. Los que se suelen dar como tales difícilmente lo son ${ }^{19}$. Son regionalismos, vulgarismos, dialectismos. ¿Por qué han de ser arcaísmos vide o truje, usados en todas las latitudes del español, desde los Balcanes hasta el Pacífico? Esos liamados arcaísmos de Canarias (aguisiar, antier, cadenado, gago, barruntar, mercar, mesmo, etc., etc.) no son ni más, ni distintos que los de cualquier otra región española.

El ESPAÑol EN UN NUEVo AMBIENTE.

Voces marineras. Palabras viejas PARA CONCEPTOS NUEVOS.

Otro problema que hay que estudiar en la dialectología canaria es el de la adaptación de una lengua a medios o ambientes distintos de los de su origen. Se han señalado en el español de América los muchos términos náuticos que en él se usan: flete ya no es 'la carga marinera', sino el 'caballo' de una u otra clase; el estero es una 'llanura' y no 'el sitio donde rajan las olas'; el rancho es 'la hacienda' y no 'el camarote de la tripulación'; etcétera, etc. ${ }^{20}$. Pero esto mismo ocurre en las islas : jalar no es sólo 'tirar de un cabo o de una cuerda', sino --también- 'llevarse la cuchara a la boca', empatar no es 'sujetar el sedal a la patilla del anzuelo', sino 'alargar, c.ñadir cualquier cosa'; liña no es el 'hilo de pescar', sino también 'la cuerda para tender la ropa', etc., etc. ${ }^{21}$. El marinerismo de estas hablas —canarias, americanas- es fácil de explicar: las largas travesías hacían que los hombres de tierra adentro se familiarizaran con la lengua de los navegntes $y$, al desembarcar, su habla - por necesidad o por broma- iba salpicada con los términos de la chusma (empleo la voz chusma en el sentido etimológico de 'dotación de una nave') y ya la lengua de tierra quedaba contaminada para siempre de la jerga marineresca.

18 Vid. Pérez Vidal, Aportación, pp. 91-127 y 181. Otros datos en Tomás NAvarro, El español de Puerto Rico. Río Piedras, 1948, p. 195.

10 Baste repasar la lista de Pedro Cullen del Castillo en Algunos arcaísmos de los subsistentes en el léxico popular canario. MCan, número 73-74, 1960, pp. 159-166.

2 Cf. A. Atonso, La base lingüistica del español americano, apud Estudios lingüisticos. Temas hispanoamericanos. Madrid, 1953, pp. 60-67, especialmente, y J. CoRominas, Rasgos semánticos nacionales. AILC, I, 1941, pp. 1-29.

I J. PÉREz VIDAL, Influencias marineras en el español de Canarias. RDTP VIII, 1952, pp. 3-25. 
Pues bien, otro aspecto de esta cuestión lingüística es el de dar nombre a las especies desconocidas. Unas veces, la imaginación vuela y ahí está el testimonio de los dragos, página abierta en cualquier libro de caballerías $2 *$. Otras veces -y son las más - se adaptan, como Dios da a entender, los viejos términos existentes. Cuando españoles y portugueses se encontraron en el Nuevo Mundo con unas frutas extrañas, las llamaron con voces de vieja cepa $y$, es curioso, recurriendo a las mismas comparaciones. En América había una fruta que podía parecerse a la piña del pino y nuestros abuelos, sin demasiados escrúpulos, la llamaron piña, mientras que los portugueses recurrieron a la voz indígena (abacaxi), porque resulta que piña se venía usando por ellos para nombrar a lo que los españoles llamaban chirimoyo o chirimoya, respetando el término autóctono.

Del mismo modo en Canarias, cuando los españoles vieron una mata olorosa la llamaron altabaca o arbeaca, en recuerdo de la alhábega o albahaca peninsular, sin darse cuenta que eran dos cosas distintas, pues la albahaca insular es la olivarda o vara de oro de Castilla ${ }^{23}$; del mismo modo, cuando vieron a la Euphorbia Canariensis pensaron en los cardos de sus pueblos y rebautizaron al cactus con el nombre de cardón, por bien distintos que sean las bellas euforbiáceas en forma de candelabro y los hierbajos pinchudos de Castilla ${ }^{24}$; otro tanto hicieron bautizando con el nombre castellano de la 'margarita silvestre' (magarza de amargaza, por su gusto) a una planta típicamente Canaria, la que los científicos llaman Leucanthemum Canariense ${ }^{25}$. Los testimonios de este tipo podrían multiplicarse ad nauseam. No merece la pena. Con los casos aducidos hay suficientes para aclarar los conceptos que he expresado en líneas anteriores ${ }^{26}$.

22 Me limito a transcribir unas líneas del Diccionario de Historia Natural de las Islas Canarias, de Viera y Clavijo (edic. 1942, t. II, pp. 254-255): (eel drago debe su celebridad a aquel su jugo propio, o resina que suda de su tronco herido en los días caniculares. Este se condensa en grumos de color de sangre... Bien sabido es que esta preciosa resina pasó mucho tiempo por una verdadera sangre de Dragón, y que el naturalista Monardes... no dudó publcar que este tal árbol había tomado la denominación de drago o de dragón por no sé qué figura de esta bestia, que parecía impresa en su fruta. Es un error, y sólo es verosímil que la húbiese tomado de la traza del tronco, rollizo y taraceado de las cicatrices de las hojas que se han caído, a semejanza del cuerpo de una gran culebra, coronado de la copa erizada como de una crestan.

23 VIERA, Diccionario, I, s. v. altavaca, pp. 61-62.

2s Op. cit., nota anterior I, pp. 171-173.

Ib., II, pp. 81-82.

* Para este apartado se consultaran con fruto las pp. 67-69 del trabajo de A Alonso citado en la nota 20. 
Las yablas Canarias, el españot. DEL SIGLO XV Y EL JUDEO-ESPAÑol.

También las islas han sufrido otra experiencia defraudadora: su lengua se ha comparado una y otra vez con el castellano de la época de los Reyes Católicos ${ }^{27}$. Pero ¿ por qué? La falsa percepción de la geografía llevó a nuarrar en lo del carácter periférico; la falsa interpretación de la nistoria lleva ahora a otros malos pasos.

Es probable que al emitir esta tesis se estuviera pensando en la crono. logía. Pero el tiempo es algo más que una hoja de almanaque irremediablemente marcada con una fecha. Es un fluir que no se remansa. $\mathrm{Y}$ bien claro el testimonio de las islas. En el siglo xv vinieron aquellos caballeros que de lina u otra forma encontraban — como el sevillano Guillén Peraza- aquí la Falma de su reposo; de ellos nacieron otros españoles, insulares ya, con los que empezó a fluir la ininterrumpida corriente de la vida hispánica de las islas. Pero estas islas no quedaron al garete de España, desarboladas sin gobernalle, sino que participaron - una región más- en los barquinazos de nuestra historia y de nuestra cultura. El español de Canarias no es un fósil de quinientos años, como un vetusto mamut siberiano, sino una jugosa realidad desde los cronistas más viejos hasta Galdós o Carmen Laforet. Y no lo es en la lengua de los grandes estilistas y no lo es tampoco en la lengua del pueblo. Aquí el español posee una indivisible unidad. Por eso Galdós tenía su libretita con palabras canarias, tan sabidas que ni siquiera se tomó el trabajo de definirlas ${ }^{28}$; por eso Carmen Laforet nos puede contar la historia, afincada en el terruño, de su majorera ${ }^{29}$. Tampoco el pueblo quedó a solas en sus islas; la vida fluyó aquí como en Sevilla o Cádiz. No creo que nadie pretenda decir que en la Puerta de Fuera, en la Alfalfa o en el Al. baicín se habla español del tiempo de los Reyes Católicos. Ni en la Vegueta o San Cristóbal, tampoco. Ahí están el yeísmo, el seseo, la aspiración de las implosivas, etc., tanto en los labios sevillanos como en los canarios. ¿Espaniol de la época de los Reyes Católicos?

Hay que desechar - también- otro espejismo: el pensar que canario y judeo-español conserven una especie de arcaísmo común ${ }^{30}$. No insisto en

27 Vid. J. Alvarez Delgado, Puesto de Canarias, pp. 19-21; Notas, pp. 207-209. Ambos trabajos se describen en las notas 7 y 11 .

* Voces canarias recopiladas por Galdós, apud Voces y frases usuales en Canarias. «Biblioteca canaria». Santa Cruz de Tenerife, s. a.

${ }^{20}$ En La Isla y los demonios. Barcelona, 1951, pp. 263 y ss.

so Vid. loc. cit. en la nota 27. 
las cuestiones geográficas e históricas, que bien asentadas quedan, pero también ahora la cronología ha cegado la claridad. No se olvide que estos judíos se incrustan en comunidades lingüísticas vivas (árabes, turcas, holandesas, griegas, búlgaras, etc., etc.) y en ellas los serardíes con minorías ciue viven al margen, en ambientes hostiles o indiferentes, defendiendo con uñas y dientes aquellas parcelas de su tesoro (la lengua, el romancero) que no quieren perder. Sin embargo, dentro de esos grandes complejos lingüísticos y culturales en los que se insertan, los sefardíes quedan aislados, sin contacto casi con la metrópoli, al menos, sin ninguna suerte de vinculación oficial. Ellos sí que son barco a la deriva, aunque sea una angustiada deriva que ya va por sus quinientos años. Por eso el judeo-español se ha ido agostando durante siglos y hoy está exhausto.

El español de Canarias no es el judeo-español. No es una lengua vencida que implora la caridad de un cobijo; es, muy al contrario, una lengua de conquista que, desde el siglo xvI ${ }^{31}$, ha eliminado las hablas prehispánicas; las relaciones de las islas con la Península son las que existen entre las regiones de cualquier territorio metropolitano; estamos -otra vez- a solas con un concepto único: el español. $\mathrm{Y}$ una variedad, otra más entre quince, de esas variedades regionales: el español de las islas Canarias.

IMPORTANCIA DE LAS HABLAS CANARIAS.

Toda esta andadura no se ha hecho para negar la personalidad del español insular; antes al contrario, para dársela y caracterizarla. Los que no nos valen son los tópicos ni las hipótesis arriesgadas. Sin embargo, hemos de aprovechar cuanto de útil encontramos al trazar nuestros proyectos. Porque ¿1 español de Canarias es, simplemente, una variedad regional de esa entidad universal que se llama el español. Su peculiaridad no está en ser una jerga incomprensible, ni un retazo perdido en la geografía, ni un andrajo maltratado por el tiempo. El español de Canarias es tan buen español y de tan buena ejecutoria como el español de cualquier otro sitio; su característica está en esos elementos con que enriquece, da variedad y hace bella a la lengua común.

El español de las islas Canarias se nos presenta, al menos en lo que sabemos, como una manifestación típica de las hablas meridionales y, dentro de

- Alvarez Delgado, Notas, p. 207, n. 4, aduce unas cuantas fuentes, pero no localiza nunca el lugar donde Torriani, Espinosa o Abreu hacen referencia a tales hechos. 
ellas, del grupo que puede llamarse atlántico. En otra ocasión he hablado de los guanchismos, arcaísmos, occidentalismos, andalucismos y americanismos que integran su léxico. Pero esta complejidad no hace sino incitarnos a un estudio demorado de las cuestiones: tanto para el conocimiento real de los hechos, cuanto para poder montar sobre ellos una especulación fundamentada. En sí, pues, la importancia de las hablas canarias es singular; pero su trajcendencia se multiplica al parangonarla con hechos que se cumplen en el єspañol de América o al considerar las islas como eslabón insoslayable entre el Viejo y el Nuevo Mundo.

Objeto de un Atlas lingüístico

de las Islas Canarias.

Acaba de publicarse el primer tomo del Atlas Lingüístico de la Península Ibérica. Obra que, si es discutible en sus planteamientos, resulta inapreciable por los materiales de conjunto que allega. Sin embargo, con una incom prensible limitación, Canarias queda fuera de la obra: ni un solo punto del Archipiélago se ha investigado allí. Y el dolor de esta ausencia es mucho mayor por cuanto ignoramos lingüística y etnográficamente casi todos los aspectos de las islas. He aquí formulada una primera urgencia: recoger los materiales para realizar, perentoriamente, el Atlas lingüístico y etnográfico de Canarias. La necesidad es inexcusable, pues gracias a él sabremos para siempre y con certeza cuáles son las peculiaridades idiomáticas del español canario, qué campos merecen una mayor urgencia de investigación, cuál es el resultado de esa larga polémica sobre la raíz - andaluza o no- del español de América, qué puesto tiene Canarias en nuestra historia lingüística, cómo fue la historia insular en todo aquello que la historia silencia. En una palabra, llenaríamos esas espaciosas lagunas que nuestros trabajos tienen cuando nos referimos a las Islas. Bástenos el recuerdo de Amado Alonso, el genial lingüista cuya muerte nunca lloraremos bastante. En uno de sus últimos y más bellos trabajos investigó la suerte de la $l l$ en español y, al llegar a las Canarias, escribía: «el yeísmo parece general, aunque faltan estudios» ${ }^{32}$ : poco después, otro gran lingüista, Juan Corominas ,al estudiar el mismo problema, incluía las islas bajo el epígrafe general de yeismo ${ }^{33}$. ¿Qué otra cosa podían hacer Amado Alonso o Corominas? Sin embargo, los hechos no son tales: las ciudades, aquí como en América o Andalucía, son yeístas; los

* La “LL" y sus alteraciones en España y América. EMP. II 1951, p. 65.

* La fecha del yeismo y del lleísmo. NRFH, VII, 1953, mapa de la p. 83. 
campos se aferran a su $l l$ y aun la mantienen durante siglos antes de percierla ${ }^{34}$. Puedo aducir algún testimonio bien significativo: una familia de pescadores de Agaete (Gran Canaria) tiene $l l$; el hijo, catedrático de lengua y literatura españolas, la ha perdido, porque las ciudades donde estudió (Las Palmas, La Laguna) abandonan el rasgo por rural. Y el yeísmo es, sólo, un botón de muestra.

El Atlas es el primer paso - no el único- que hay que dar. Es el primer paso porque es obra hacedera, porque en ella deben trabajar muy pocos operarios. porque los frutos granan día a día. Bien entendido, los Atlas han revolucionado la ciencia lingüística en medida que ningún otro método ha conseguido hacerlo. Nuestro retraso frente a las demás naciones es porque hasta 1961 no tuvimos un Atlas del dominio castellano y ahora, cuando se aseguran nuevas empresas, el poder igualatorio de nuestro tiempo va haciéndonos ver todo lo tardíamente que España se ha incorporado a la cartografía lingüística. Pero no debemos caer en un falso espejismo: un Atlas no es una panacea universal. Es, simp'emente, un espléndido instrumento de trabajo; no hay gue pedirle lo que no puede dar. El Atlas es una obra limitada: investiga -sólo- unos cuantos puntos y pregunta - sólo- unas cuantas cuestiones. El director de la obra elige los lugares que se van a investigar y, previamente, ordena el Cuestionario común que estima más conveniente. A pesar de estas limitaciones, los frutos son generosos. Baste -como he hecho otras vecesın grosero comentario. Pensemos en nuestras is'as. Unos cincuenta puntos estudiados harían de Canarias la región más densamente investigada de toda la Romania: desde las bocas del Danubio hasta el Pacífico ${ }^{35}$. Usando un Cuestionario de unas 1.300 preguntas, podremos obtener inmediatamente ese inmediatamente que es el final de la obra, unas sesenta y cinco mil formas. Naturalmente, hay muchas repeticiones. pero ¿podemos pensar qué serán esas 65.000 formas para la lingüística insular si pensamos que están ordenacias geográficamente, rigurosamente transcritas, extendidas sobre todos los aspectos de la vida humana? Valga una sola referencia, que otras veces he aducido: el Diccionario de la Academia registra unas 73.000 voces, según el cómputo de don Julio Casares ${ }^{36}$.

En Andalucía hemos recogido como medio millón de formas, de las que ya hay publicadas en mapas más de la mitad. ¿Por qué una empresa seme-

34 Vid. $\$ 32$, pp. 40-42 de mi Español de Tenerife.

${ }_{35}$ Para esto, cf. M. Alvar, El Atlas Lingüistico-Etnográfico de Andalucí x. PALA, I, número 4, 1959, p. 21, y Metodología e historia lingüistica. A propósito del Attlas de Rumania. «Acta Salmanticensia», IV, 1951, pp. 13-14.

so Vid. PALA, I, cit. nota anterior, p. 9. 
jante no se puede hacer en cualquier región de España? Y Andalucía es la mayor, la más poblada, la más difícil lingüísticamente hablando.

Esas 65.000 formas del futuro Atlas Canario constituyen un material inmenso. El Léxico de Gran Canaria de los hermanos Millares tiene unas 520 voces. Modestísima cantidad, incluso para un léxico, pues Baráibar recogió 1.100 voces alavesas; García Rey, 2.600 bercianas; Borao, unas 3.500 aragonesas; Lamano, unas 4.000 salmantinas; Alcalá, unas 16.000 anda luzas $^{37}$. Y me permito insistir en un hecho: el caudal léxico es sólo una parte $\rightarrow$ y no la más trascendental - que el Atlas podría aportar.

Pero, ya lo he dicho, el Atlas no es una panacea. Es necesario espesar su red, investigar los campos que deja en barbecho, estudiar la estratigarfía social del dialecto, etc., etc. En una palabra: investigar monográficamente. Uno de los más grandes romanistas de todos los tiempos, Gaston Paris, dijo que hace falta disponer de la descripción de cada habla local hecha con rigor y de primera mano ${ }^{38}$. Es necesario aspirar a ello. Pero la lengua es sutil y cambiante. La monografía tendría que repetirse cada dos o tres generaciones y entonces veríamos - lo hemos visto en otros dos dominioscómo la fonética, las preferencias léxicas, todo ese delicado instrumento que es el habla viva, cambia de padres a hijos. Nosotros no podemos aspirar más que a dar idea de nuestra limitación: obtener una instantánea lingüística en un momento determinado. Esto que parece tan poca cosa ha de ser nuestro trabajo durante una década cuando menos.

\section{I.os funtos de encuesta.}

La superficie de las Islas Canarias y su población es la siguiente (Censo de 1960):

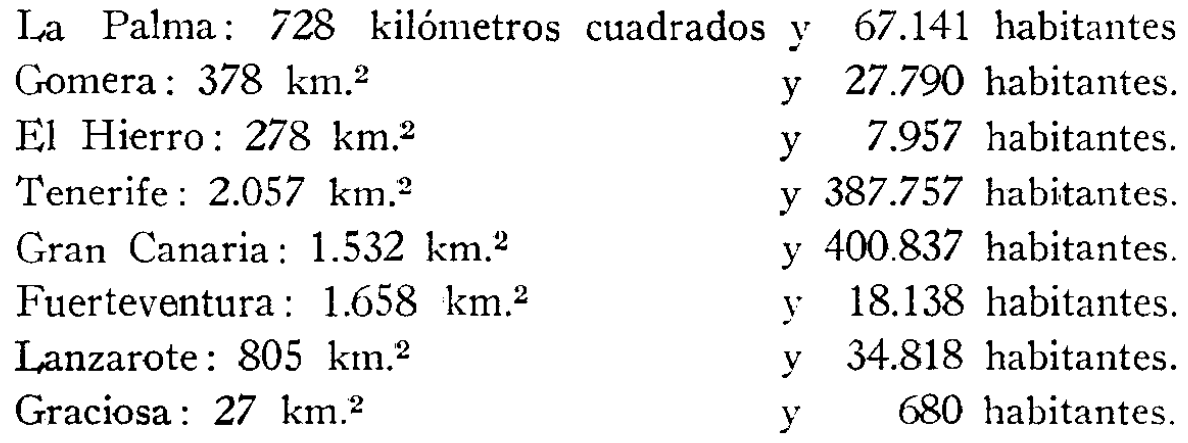

$\$$ Les parlers de France. RPGR, II, 1888, p. 168.

y El Atlas, ya cit., p. 10, nota 26.

? Datos de mi Atlas lingiuistico de Andalucía. Cuestionario. Granada, 1952, p. V, 
Para este total de 7.463 kilómetros cuadrados y 945.118 habitantes se proyecta el estudio de unas 50 localidades ${ }^{39}$. En principio, y de una forma puramente teórica, se han distribuído 45 puntos sobre la superficie de las islas. E1 explorador podrá seleccionar, sobre el terreno, aquellos puntos que sean más idóneos. En esto seguimos el mismo prudente criterio que Jud y Iaberg implantaron para el AIS y que, sin excepción, venimos practicando cuantos nos dedicamos a las encuestas dialectales. Bien entendido, que se adensará la red cuando el interés lingüístico así lo exija y bien entendido, también, que la lista siguiente es -sólo- orientadora y en modo alguno definitiva.

\section{Puntos de encuesta.}

La Palma: Santa Cruz de la Palma, El Paso, Fuentecaliente, Tijarafe, Garafía, San Andrés.

Gomera: San Sebastián, Vallehermoso, La Calera, Alajeró (Santiago).

Fín Hierro: Valverde, Sabinosa, Taibique, San Andrés.

'Tenerife: Santa Cruz de Tenerife, Taganana, La Laguna, La Orotava,

Buenavista, Boca de Tauce, Granadilla, Los Cristianos, El Médano, Arico el Viejo, Isa Candelaria, Güimar.

Gran Canaria: Las Palmas, Guía, Teror, Agaete, Artenara, San Nicolás, San Bartolomé de Tirajana, Mogán, Agüimes, Arguineguín.

Fuerteventura: Puerto del Rosario, Betancuria, Puerto de Gran Tarajal, Corralejo.

Lanzarote: Arrecife, Femés, Orzola,Tinajo.

Graciosa: Caleta del Sebo.

30 Así, pues, en Canarias investigaremos un punto cada 156 kilómetros cuadrados y 1.890 habitantes, mientras que en Andalucía lo hicimos cada $380 \mathrm{Km}$. $24.348 \mathrm{~h}$. y en Aragón cada 432 y 9.945, respectivamente. (Vid., para estas últimas cifras, el Proyecto de un "Atlas Lingüistico y Etnográfico de Aragón». Anejo VIII del AFA. Zaragoza, 1963, p. 13.) Los datos recién transcritos de Andalucía son definitivos y adensan los que dí, al preparar la obra, en el Proyecto de un Atlas Lingüistico de Andalucía. "Orbis», III, 1951, p. 55. Como se ve, el espesor de la red es superior al de cualquier otra obra semejante (compárese con los datos que doy en los trabajos, ya citados, Metodología, pp. 13-14, y Proyecto, p. 55. nota 1). 


\section{El Cuestionario.}

El Cuestionario se ha redactado tomando como base el del ALEA ${ }^{40}$. Ahora bien, la experiencia de seis encuestas que hice en la isla de Tenerifet me permitió redactar un nuevo cuestionario de 1.800 prguntas, que presenté al Consejo Superior de Investigaciones Científicas, junto a un primer proyecto de Atlas regional ${ }^{42}$. Sin embargo, en el verano de 1963 llevé a cabo unas minuciosísimas encuestas en la isla de Gran Canaria (Teror y Las Palmas), a la vez que investigué con sumo cuidado la terminología marinera de las playas de Las Canteras y San Cristóbal (Las Palmas). Estos nuevos informes me permitieron reestructurar el cuestionario y darle la forma definitiva que ahora tiene. Así, pues, sobre un original (ALEA) he ido elaborando mis preguntas conforme adquiría un mejor conocimiento de la realidad regional (encuestas en Tenerife y Gran Canaria); al tiempo que hacía otras innovaciones que me parecieron oportunas tras haber redactado el cuestionario del ALEAR ${ }^{43}$. Me parece obvio decir que he dado una importantísima cabida a las cuestiones típicamente insulares (vegetación, cultivo del plátano, nomenclatura del camello, vasijas, etc., etc.) para apurar - en cuanto sea posiblela originalidad regional. Pero he tratado, a toda costa, de mantener la unidad de esta investigación con otra ya emprendidas: si la Asamblea de Instituciones Hispánicas celebrada en Madrid (junio de 1963) recomendó el uso de los Cuestionarios del ALEA y del ALEAR para cualquier futuro $\Lambda$ tlas del dominio Hispánico ${ }^{44}$, no podíamos desentendernos de ello al proyectar el estudio de una región del español meridional.

En cuanto al $\$ 24$ (El mar, etc.), se ha hecho tomando como base los dos tomos del Questionario dell'Atlante Linguistico Mediterraneo, de la Fonclazione Giorgio Cini (Venecia, s. a.) ${ }^{45}$. Los resultados positivos que he obtenido, junto a otros informes de investigación personal, se resumen en las

to Sobre él se pueden leer mis notas en las págs. X-XVI que puse a su prólogo, y las pp. 13-20 de El Atlas citado en la nota 35. Existe un antiguo Proyecto de cuestionario del Folk-lore canario debido a Juan de Béthencourt Alfonso, e incluido en las pp. $20 b-22 b$ y $29 a-b$ de los números 3 y 4 del Bocltín folklórico español.

11 Vid. mi Español hablado en Tenerife, pp. 9-11.

13 Ib., p. 4.

43 Cf. Proyecto de un Atlas de Aragón, pp. 18-22.

${ }^{44}$ Vid. Instituto de Cultura Hispánica, Congreso de Instituciones Hispánicas. Madrid, 1964, pp. 115-116.

45 He hecho con esos cuestionarios todas las encuestas del Sur y Sureste de España y de nuestras Plazas africanas que figurarán en el ALM. Además, he traducido los dos volúmenes de que consta la obra y he compulsado sus datos con obras españolas de náutica e ictiología. 
de la FAO, Roma, 1960, y A. Palombi-M. Santarelli, Gli animali commestibili dei mari d'Italia (2. ${ }^{\mathrm{a}}$ edic.), Milán, 1960. Estos tres libros unen la bondad de sus gráficos a la comodidad de su manejo, incluso en condiciones poco cómodas. No creo que el número de preguntas marineras pueda parecer excesivo si pensamos en el Archipiélago que vamos a investigar; pero hay cue añadir otras cuestiones: el descuido que de la lengua de los pescadores con el tomo de láminas del Questionario y con las ilustraciones de las obras siguientes: L. Lozano, Los principales peces marinos y fluriales de España (2. ${ }^{\mathrm{a}}$ edic.), Madrid, 1949; el Catalogue de Nomes de Poissons/Fish Names preguntas 1.148-1.341 que ahora presento. Mis búsquedas in situ se ayudaron se ha hecho en otros Atlas; el proyecto del ALM y mi presensión de llevar a cabo una obra de cartografía lingüística tomando como base el léxico marinero peninsular.

\section{LA REALIZACIÓN DE LA OBRA.}

En el momento de corregir estas pruebas (14 de junio de 1965) se han llevado a cabo las siguientes encuestas:

Graciosa: Caleta del Sebo (investigación doble, con un marinero y una mujer).

LANZAROTE: Arrecife (encuesta doble, con marinero y labrador), Fenés, María (en Orzola, pueblecito de pescadores, se investigó la parte marinera del cuestionario), Tiagua (ofrecía un interés etnográfico mucho mayor que Tinajo, y está muy cerca de él).

Gran Canaria: Las Palmas (y dos encuestas con pescados, a las que ya he hecho mención). Guía, Teror, Agaete, Artenara, San Nicolás de Tolentino, San Bartolomé de Tirajana, Mogán (y otra encuesta complementaria muy extensa en Puerto de Mogán), Agüimas y Arquineguín.

El Instituto de Estudios Canarios (C. S. I. C.) patrocina las encuestas. De momento actuaré como explorador único y creo que en un plazo de tres o cuatro años las habré podido completar. Si la obra llega a buen fin, la lingüística española se kabrá enriquecido con inéditos y hermosos aspectos. Si no hubiera tras el trabajo un alto interés científico, bien valdría la pena llevarlo a cabo como homenaje a las sietc islas, bellas como los siete rayos del espectro solar.

Manuel Alvar. 